\title{
CONDICIONAMENTO FISIOLÓGICO DE SEMENTES DE CHICÓRIA COM Ascophyllum nodosum
}

\author{
Amanda Ferraz ${ }^{1}$, Vanessa Neumann Silva ${ }^{2 *}$, André Luiz Radunz ${ }^{3}$ \\ ${ }^{1}$ Graduanda do curso de Agronomia, Universidade Federal da Fronteira Sul, campus Chapecó, Santa Catarina. \\ ${ }^{2}$ Doutora em Fitotecnia, Professora Adjunta, Universidade Federal da Fronteira Sul, campus Chapecó, Santa \\ Catarina. *E-mail do autor correspondente: vanessa.neumann@uffs.edu.br \\ ${ }^{3}$ Doutor em Agronomia, Professor Adjunto, Universidade Federal da Fronteira Sul, campus Chapecó, Santa \\ Catarina.
}

Recebido: 14/12/2018; Aceito: 04/07/2019

RESUMO: Sementes de chicória podem apresentar baixo potencial germinativo, o que traz problemas ao olericultor. Sendo assim, procedimentos que possam promover a melhoria do potencial da semente, como o condicionamento fisiológico, necessita ser estudado. O objetivo deste trabalho foi verificar efeitos do condicionamento fisiológico das sementes de chicória com bioestimulante de Ascophyllum nodosum na germinação e produção de mudas. $\mathrm{O}$ experimento foi conduzido em delineamento experimental inteiramente casualizado, com esquema fatorial $2 \times 4$ (cultivares e doses). Foram utilizadas sementes de chicória das cultivares Lisa e Crespa, e as doses de 0, 5, 10 e $20 \mathrm{~mL}$ de bioestimulante $\mathrm{kg}-1$ de sementes. Inicialmente foram realizadas curvas de embebição para definição da duração do condicionamento. Posteriormente, as sementes foram condicionadas e submetidas as seguintes avaliações: porcentagem e velocidade de germinação, comprimento de plântulas em laboratório, porcentagem e velocidade de emergência de plântulas, altura de mudas, número de folhas e comprimento de raízes em casa de vegetação. Os resultados obtidos foram submetidos à análise de variância e à análise de regressão (doses) e teste de Tukey (cultivares). A germinação de sementes de chicória aumenta linearmente com o aumento de doses de Ascophyllum nodosum via condicionamento de sementes. O condicionamento de sementes de chicória com Ascophyllum nodosum contribui na fase inicial de produção de mudas, com incrementos na porcentagem de emergência de plantas aos sete dias após a semeadura, assim como na velocidade de emergência, porém, esse efeito não persiste posteriormente. A reposta ao condicionamento de sementes de chicória com Ascophyllum nodosum é variável em função da cultivar utilizada.

Palavras-chave: Cichorium endivia. Bioestimulante. Alga marrom.

\section{CICHORIUM SEED PRIMING WITH Ascophyllum nodosum}

ABSTRACT: seeds of chicory, can present, low germinative potential, which brings problems to horticultural farms. Thus, procedures that can promote the improvement of seed potential, such as seed priming, need to be studied. The objective of this work was to evaluate the effects of the chicory seed priming with Ascophyllum nodosum biostimulant on germination and seedling production. The experiment was conducted in a completely 
randomized design, with a $2 \times 4$ factorial scheme (cultivars and doses). Chicory seeds of the cultivars Lisa and Crespa, and the doses of $0,5,10$ and $20 \mathrm{ml}$ of biostimulant $\mathrm{Kg}^{-1}$ of seeds were used. Initially, seeds were submitted to imbibition curves test to define priming duration procedure. Chicory seeds were conditioned and submitted to evaluations of: percentage and germination speed, and length of seedlings in the laboratory, percentage and emergence speed of seedlings, seedling height, number of leaves and length of roots in greenhouse. The results were submitted to analysis of variance and regression analysis (doses) and Tukey test (cultivars). The germination of chicory seeds increases linearly with increasing doses of Ascophyllum nodosum via seed conditioning. The conditioning of chicory seeds with Ascophyllum nodosum contributes to the initial phase of seedling production, with increases in the emergence percentage of plants at seven days after sowing, as well as at the emergence speed, but this effect does not persist subsequently. The response to the conditioning of chicory seeds with Ascophyllum nodosum varies according to the cultivar used.

Key words: Cichorium endivia. Bioestimulant. Brown seaweed.

\section{INTRODUÇÃO}

A rápida e uniforme germinação e emergência é condição importante para o estabelecimento de plantas, em sistemas de cultivos agrícolas. Sementes de hortaliças, em sua maioria, são de tamanho reduzido e contém pequena quantidade de reservas nutritivas, em seus tecidos, geralmente fator que leva a desuniformidade na germinação.

Alguns resultados de pesquisas indicam que tratamentos de sementes podem contribuir para melhoria da qualidade das mesmas, que contribuem para o desempenho fisiológico de sementes. Entre os tratamentos, está o condicionamento fisiológico. De acordo com Paparella et al. (2015), o condicionamento fisiológico permite a hidratação controlada de sementes para desencadear os processos metabólicos normalmente ativados durante a fase inicial da germinação ("metabolismo pré-germinativo"), mas impedindo a transição de sementes para a germinação total; é uma técnica que permite a melhoria da qualidade de sementes.

O condicionamento de sementes pode trazer uma série de benefícios, como germinação rápida e uniforme, redução da foto e termo-dormência, maior adaptação a amplitude de temperaturas de germinação e melhor capacidade de competir com sementes de plantas daninhas e patógenos (ELLIS; BUTCHER, 1988).

Atualmente, já se tem estudos referentes à eficácia desta técnica, com sementes de hortaliças, tais como: cenoura (LOPES et al., 2011), pimentão (SILVA et al. 2015), rabanete (ASHRAF et al., 2018), cebola (ZHAO et al., 2018), quiabo (SHARMA et al., 2014) entre outras. Segundo Paparella et al. (2015) já existe uma série de protocolos de condicionamento patenteados, obtidos por empresas de tratamento de sementes como por exemplo: EasyPrime ${ }^{\circledR}$ e EasyDormex ${ }^{\circledR}$ da empresa ATLAS (Itália) para sementes de tomate, pimentão, berinjela, melão e brássicas; Advantage ${ }^{\circledR}$, Xbeet ${ }^{\circledR}$ e Emergis ${ }^{\circledR}$ da empresa Germains Seed Technology, para sementes de beterraba, flores e várias hortaliças, respectivamente; Thermocure $^{\mathrm{TM}}$, Splitkote ${ }^{\circledR}$ Special, Promotor ${ }^{\mathrm{TM}}$, Improver ${ }^{\mathrm{TM}}$, da empresa INCOTEC (Holanda), para sementes de alface, chicória, almeirão, cebola, cenoura, tomate e brássicas. 
Em estudos realizados com sementes de chicória foi constatada a eficácia do condicionamento fisiológico com ácido ascórbico, quanto a porcentagem de germinação e crescimento de mudas, assim como para tolerância ao estresse hídrico (SADEGI; ROBATI, 2015).

Sendo assim, a técnica de condicionamento têm eficiência comprovada em muitas pesquisas, especialmente para sementes de hortaliças, contudo, não existem ainda tecnologias brasileiras estabelecidas e tão pouco estudos dessa técnica com produtos permitidos na agricultura orgânica, especialmente que envolvam o uso de algas. Considerando o aumento expressivo do mercado de produtos orgânicos e a necessidade de insumos para sua produção, especialmente de hortaliças, fazem-se necessários mais estudos envolvendo essa temática.

Diante disso, o presente trabalho, objetivou verificar os efeitos do condicionamento fisiológico de sementes de chicória com bioestimulante à base de Ascophyllum nodosum na germinação e produção de mudas de chicória.

\section{MATERIAL E MÉTODOS}

O experimento foi conduzido em Laboratório e em Casa de Vegetação. O delineamento experimental empregado foi o inteiramente casualizado, em esquema fatorial 2 x 4 (cultivares e doses). Foram utilizadas sementes das cultivares Escarola Lisa e Crespa, e quatro doses de bioestimulante via condicionamento fisiológico: 0 (controle), 5,10 e $20 \mathrm{mg} \cdot \mathrm{kg}^{-1}$ de sementes (SOUZA NETA, 2016).

Inicialmente foi realizada a curva de embebição, para todas as doses de bioestimulantes utilizando-se duas repetições de $0,10 \mathrm{~g}$ de sementes de Chicória, de cada cultivar, separadamente, as quais foram distribuídas entre três folhas de papel toalha, umedecidas com água a uma proporção 2,5 vezes a massa do substrato seco; a seguir foram colocadas em germinadores regulados a temperatura de $20^{\circ} \mathrm{C}$. As pesagens foram realizadas em intervalos de 1 hora até 24 horas; posteriormente, foram efetuadas pesagens em intervalos de duas horas, onde as sementes foram retiradas do papel toalha, secas superficialmente com papel filtro, pesadas e colocadas novamente para embeber, o procedimento foi conduzido até a protrusão da raiz primária de $10 \%$ das sementes conforme Ferreira et al. (2013).

O condicionamento foi realizado durante 23 horas, definido a partir da análise dos resultados da curva de embebição, utilizando as doses de $0,5,10$ e $20 \mathrm{~mL}$ de bioestimulante $\mathrm{kg}^{-1}$ de sementes (SOUZA NETA, 2016). Na sequência foram realizados os testes para avaliação do efeito do tratamento, conforme descrição:

Germinação: foi realizada utilizando-se quatro repetições de 50 sementes, distribuídas sobre duas folhas de papel (germitest ${ }^{\circledR}$ ) umedecidas com quantidade de água equivalente a 2,5 vezes a massa seca do papel, dispostos em caixas plásticas tipo gerbox. O conjunto foi exposto a temperatura de $20^{\circ} \mathrm{C}$, em câmara de germinação, com fotoperíodo ajustado para 8 horas de luz e 16 horas de escuro (BRASIL, 2009). As avaliações foram realizadas de acordo com os critérios estabelecidos nas Regras para Análise de Sementes e os resultados foram expressos em porcentagem de plântulas normais para cada tratamento (BRASIL, 2009). 
Índice de velocidade de germinação: foi determinada em conjunto com o teste de germinação, com contagens diárias, contabilizando-se o número de sementes germinadas e utilizando-se a fórmula proposta por Maguire (1962) para o cálculo do índice.

Comprimento de plântulas: As avaliações foram realizadas em 20 plântulas por repetição, retiradas aleatoriamente de cada tratamento, a partir do teste de germinação. As plântulas foram medidas com régua graduada e os resultados foram expressos em $\mathrm{cm}$ plântula-1 (NAKAGAWA, 1999).

A segunda etapa da pesquisa para verificar o efeito do condicionamento fisiológico na produção de mudas, foi realizada de acordo com a seguinte metodologia:

Emergência de plantas: Quatro repetições de 50 sementes foram distribuídas em bandejas de 128 células (22,5 $\mathrm{ml}$ por célula), com substrato comercial para hortaliças composto de turfa e casca de arroz, dispostas sobre bancada, com irrigação por aspersão. Aos sete, 14, 21 e 28 dias após a semeadura (DAS) foram avaliados porcentagem de emergência de plântulas, altura de mudas e número de folhas, seguindo metodologia de Silva et al. (2017) em 20 plantas, aleatoriamente, de cada repetição. Aos 28 DAS avaliou-se o comprimento de raízes de 20 plantas retiradas do substrato, aleatoriamente, lavadas em água e secas com papel toalha, e posteriormente realizada a medição com régua graduada, conforme SILVA et al. (2017). O cálculo do índice de velocidade de emergência de plantas foi realizado a partir de contagens diárias de plantas emersas, até valor constante, a partir da emergência da primeira planta, seguindo a fórmula de Maguire (1962).

Os dados obtidos no experimento foram submetidos à análise de variância $(\mathrm{p}<0,05)$ e quando o teste $\mathrm{F}$ foi significativo procedeu-se à análise de regressão para o fator doses e o teste de médias Tukey para o fator cultivares.

\section{RESULTADOS E DISCUSSÃO}

As curvas de embebição de sementes de chicória mostram padrão diferencial de absorção de água/solução em função dos tratamentos utilizados, tanto para cultivar Crespa (Figura 1A), quanto para a cultivar Lisa (Figura 1B).

É importante destacar que no tratamento testemunha observou-se protrusão de raiz nos períodos de 31 e 29 horas de embebição, para as cultivares Crespa e Lisa, respectivamente; entretanto, quando se utilizou bioestimulante, esse tempo diminuiu, ficando entre 26 e 27 horas para a cultivar Crespa e 27 horas para a Lisa. É provável que o bioestimulante tenha acelerado as etapas iniciais de germinação, permitindo a protrusão de raiz em tempo menor.

Posteriormente à realização das curvas de embebição foi definido o tempo de 23 horas para o condicionamento ser realizado, pelo fato de que a partir de 24 horas alguns tratamentos já apresentavam algumas sementes germinadas, principalmente nos tratamentos com bioestimulante, pois este, segundo Marcos Filho (2015), faz com que as sementes acelerem seu metabolismo germinativo e o condicionamento fisiológico deve ser interrompido antes da protrusão da raiz primária. 
A

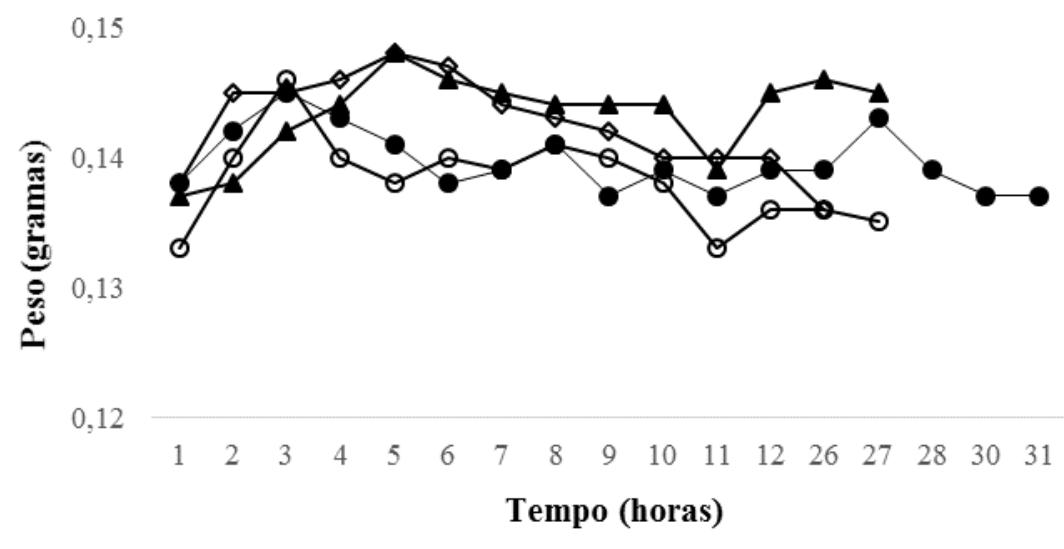

B

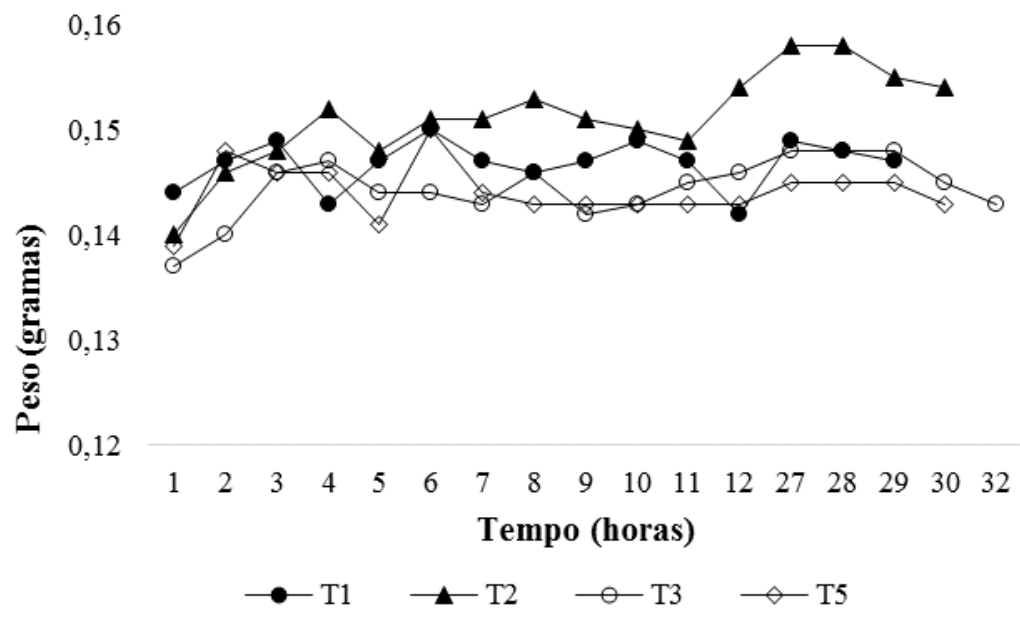

Figura 1. Curvas de embebição de sementes de chicória, cultivares Crespa (A) e Lisa (B), condicionadas com doses de $0(\bullet), 5(\boldsymbol{\Delta}), 10(\circ)$ e $20(\diamond) \mathrm{mg}$ de bioestimulante $\mathrm{Kg}^{-1} \mathrm{de}$ sementes. Curves of chicory seeds imbibition, cultivars Crespa (A) and Lisa (B), primed with doses of $0(\bullet), 5(\mathbf{\Delta}), 10(0)$ and $20(\diamond) \mathrm{mg}$ of biostimulant $\mathrm{Kg}^{-1}$ of seeds.

Em relação aos efeitos do condicionamento, para as variáveis porcentagem (Figura 2A) e velocidade (Figura 2B) de germinação, observou-se diferença entre doses, com aumento linear em função da dose utilizada, possivelmente pela ação das substâncias estimulantes presentes na alga marrom.

Segundo Battacharyya et al. (2015) vários trabalhos de pesquisa já confirmaram a presença de reguladores de crescimento em extratos de alga marrom, como ácido indol acético, citocininas, ácido giberélico, poliaminas e ácido abscísico (PROVASOLI; CARLUCCI, 1974; STIRK et al., 2003; KHAN et al., 2009; WALLY et al., 2013), os quais regulam vários processos fisiológicos nas sementes, incluindo a germinação (MIRANSARI; SMITH, 2014). 
A

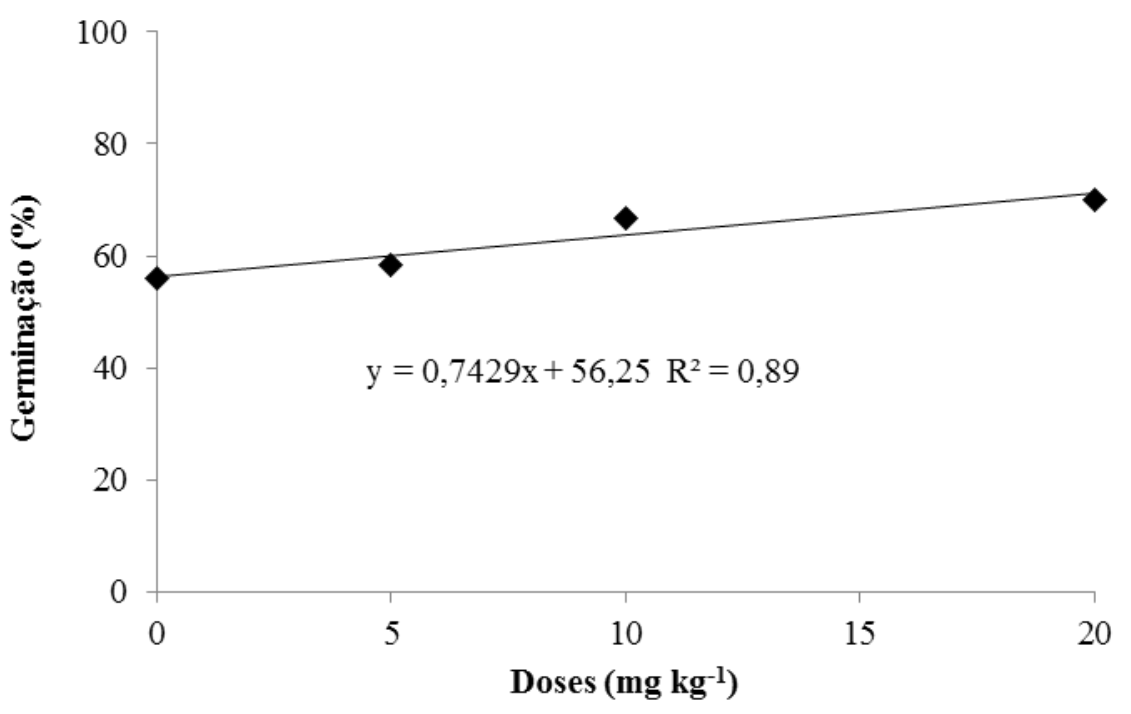

B

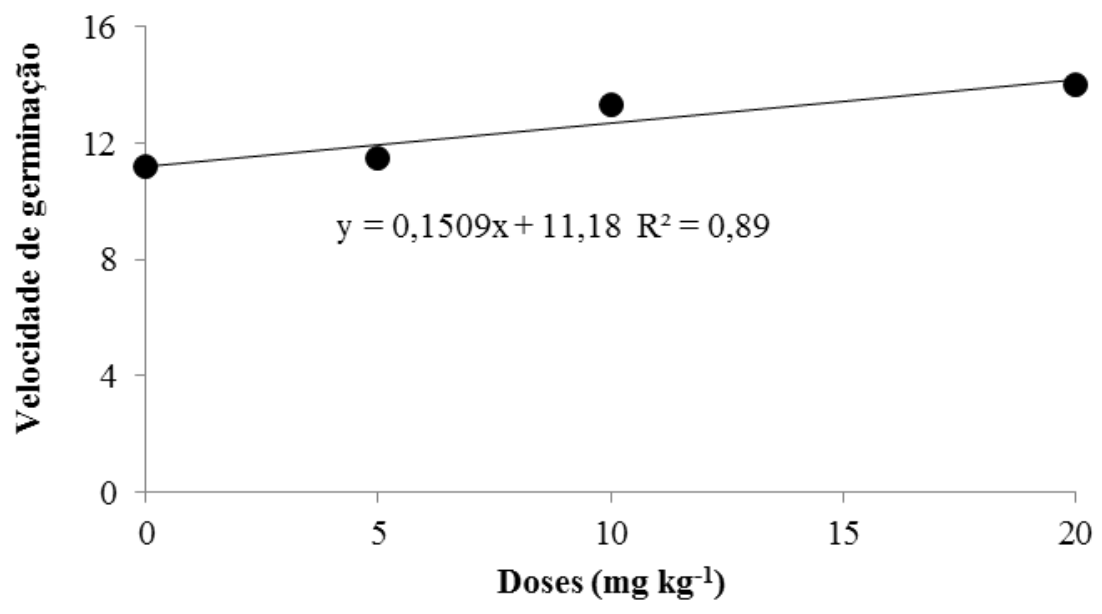

Figura 2. Germinação (A) e velocidade de germinação (B) de sementes de chicória condicionadas com diferentes doses de bioestimulante. Germination (A) and germination speed index $(B)$ of chicory seeds primed with different doses of biostimulant.

Resultados semelhantes, com o incremento da germinação de sementes, em função do uso da alga marrom no condicionamento fisiológico foram verificados para tomate (SIVRITEPE; SIVRITEPE, 2016) e lavanda (DEMIRKAYA et al., 2017).

Quanto ao comprimento de plântulas observou-se apenas diferenças entre as cultivares (Tabela 1), com maior desempenho da cultivar Crespa em todos os tratamentos com bioestimulante. Vale ressaltar que, embora o fator dose não tenha diferido estatisticamente, na Cultivar Crespa houve aumento de $1,8 \mathrm{~cm}$ no comprimento de plântula, entre o tratamento testemunha e as doses de 5 e $10 \mathrm{~mL} \mathrm{~kg}^{-1}$, o que pode ser interessante para acelerar o crescimento da planta e reduzir o tempo necessário para produção de mudas. 
Tabela 1. Valores médios de comprimento de plântulas (CP) de chicória, aos 14 DAS obtidas de sementes condicionadas com diferentes doses de bioestimulante. Mean values of seedling length $(C P)$ of chicory at 14 DAS obtained from seeds conditioned with different doses of biostimulant.

\begin{tabular}{ccccc}
\hline \multirow{3}{*}{ Cultivar } & \multicolumn{5}{c}{ Doses $\left(\mathrm{mg} \mathrm{Kg}^{-1}\right.$ de sementes $)$} \\
\cline { 2 - 5 } & \multicolumn{4}{c}{5} \\
\cline { 2 - 5 } & $4,68 \mathrm{a}^{*}$ & $3,35 \mathrm{~b}$ & $1,73 \mathrm{~b}$ & 20 \\
\hline Lisa & $4,44 \mathrm{a}$ & $6,24 \mathrm{a}$ & $6,24 \mathrm{a}$ & $5,9 \mathrm{a}$
\end{tabular}

*Médias seguidas de mesma letra na coluna, não diferem entre si pelo teste de Tukey $(\mathrm{p}<0,05)$. *Means followed by the same letter in the column, do not differ by Tukey test $(p<0,05)$.

Em relação ao efeito do condicionamento de sementes de chicória na emergência de plantas observou-se não haver diferenças entre as cultivares. Porém, efeito de doses ocorreu para a porcentagem de emergência de plantas aos sete dias após a semeadura (DAS) (Figura 3A) e velocidade de emergência de plantas (Figura 3B), com resposta quadrática. Maior desempenho para essa variável foi observado na dose de $13,1 \mathrm{~mL} \mathrm{~kg}^{-1}$ de sementes. Entretanto, aos 14, 21 e 28 DAS (Tabela 2) não foram constatados efeitos do condicionamento, pois, provavelmente não tenha persistido nessas datas de avaliações, tendo efetividade apenas na primeira semana após o tratamento das sementes, para essas características. Entretanto, ao melhorar a emergência na fase inicial, essa técnica pode contribuir para bom desempenho das sementes, na produção de mudas.

Sadegi e Robati (2015) em estudo sobre métodos para condicionamento osmótico de sementes de chicória, verificaram que o procedimento proporcionou aumento na capacidade de germinação em sementes submetidas à situação de estresse hídrico, e, portanto, nesta pesquisa, o tratamento com bioestimulante com Ascophyllum nodosum alcançou importância prática, sugerindo que pode ser recomendado para se obter maior germinação e emergência uniforme em campo, especialmente em sementes submetidas a essas condições.

Em relação à altura de mudas, observou-se diferenças apenas entre as cultivares aos 14, 21 e 28 DAS, com menor desempenho das plantas provenientes da cultivar Lisa, no tratamento sem bioestimulante (Tabela 2).

Aos sete DAS não houve diferenças entre cultivares e doses. De forma análoga a esse resultado, para a variável número de folhas, não houve diferenças entre as plantas das cultivares Lisa e Crespa e doses utilizadas no condicionamento (Tabela 2). 

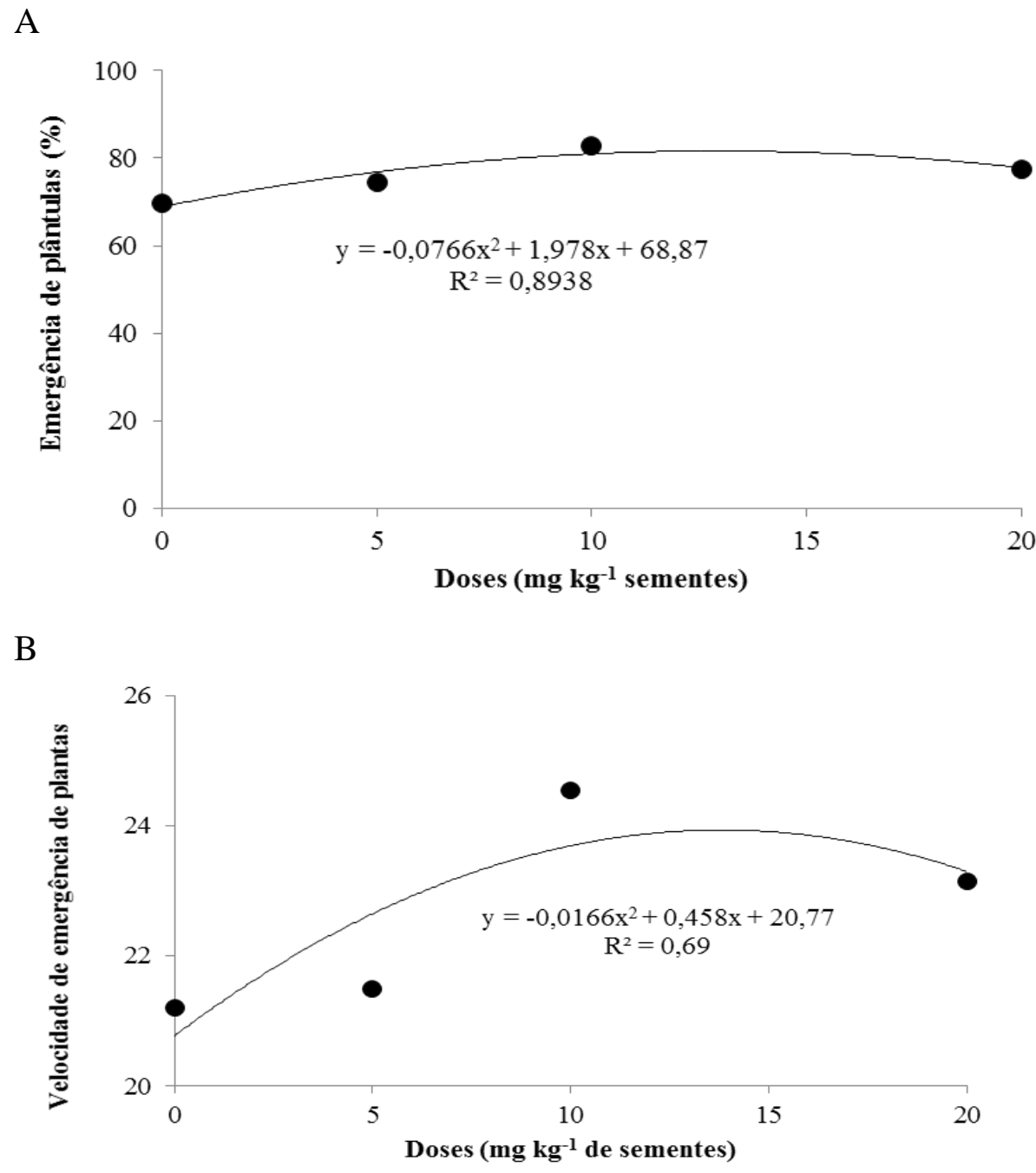

Figura 3. Valores médios de porcentagem de emergência (A) e de velocidade de emergência de plantas (B) de chicória, aos sete dias após a semeadura, em função de diferentes doses de Ascophyllum nosodum, via condicionamento de sementes. Mean values of emergence percentage (A) and speed plant emergence index (B) of chicory, seven days after sowing, as a function of different doses of Ascophyllum nosodum, via seed priming.

Os valores encontrados para essas variáveis ficam próximos aos verificados por outros autores, sem tratamento das sementes, como no trabalho de Cardoso e Ustulin Filho (2013), os quais constataram valores variando entre 3,9 a $5,5 \mathrm{~cm}_{\text {planta }}{ }^{-1}$, para altura de mudas e de 4 a 6 para número de folhas de chicória, cultivar Malan. Contudo, cabe destacar que no trabalho dos referidos autores, as mudas foram avaliadas aos 43 DAS, período superior a presente pesquisa e com outra cultivar.

Quanto ao comprimento de raízes de plantas, observou-se diferença para as raízes da cultivar Lisa provenientes do tratamento testemunha e $20 \mathrm{~mL} \mathrm{~kg}^{-1}$ de sementes (Tabela 2).

Quanto ao uso de diferentes doses de Ascophyllum nodosum, assim como na presente pesquisa, Foekel et al. (2015) constataram não haver efeito do tratamento de sementes de maracujá com Acadian ${ }^{\circledR}$ (fabricado a partir da alga Ascophyllum nodosum) na emergência, altura da planta, número de folhas e no crescimento radicular de plantas. 
Tabela 2. Valores médios de emergência de plantas (EP), altura de mudas (AM), número de folhas (NF) de chicória, aos sete, 14, 21 e 28 dias após a semeadura (DAS) e comprimento de raízes de plantas (CRP) aos 28 DAS, obtidas de sementes condicionadas com diferentes doses de bioestimulante. Mean values of emergence of plants (EP), height of seedlings (AM), number of leaves (NF) of chicory, at seven, 14, 21 and 28 days after sowing (DAS) and plant root length (CRP) to 28 DAS, obtained from seeds conditioned with different doses of biostimulant.

\begin{tabular}{|c|c|c|c|c|}
\hline \multirow[t]{2}{*}{ Cultivar } & \multicolumn{4}{|c|}{ Doses de bioestimulante ( $\mathrm{mg} \mathrm{Kg}^{-1}$ de sementes) } \\
\hline & 0 & 5 & 10 & 20 \\
\hline & \multicolumn{4}{|c|}{ EP 14 DAS (\%) } \\
\hline Lisa & $74,5^{\mathrm{NS}}$ & 74,0 & 82,5 & 82,5 \\
\hline \multirow[t]{2}{*}{ Crespa } & 70,0 & 79,5 & 82,5 & 74,0 \\
\hline & \multicolumn{4}{|c|}{ EP 21 DAS (\%) } \\
\hline Lisa & $74,5^{\mathrm{NS}}$ & 74,0 & 82,5 & 82,5 \\
\hline \multirow[t]{2}{*}{ Crespa } & 71,5 & 80,0 & 82,5 & 74,0 \\
\hline & \multicolumn{4}{|c|}{ EP 28 DAS (\%) } \\
\hline Lisa & $74,5^{\mathrm{NS}}$ & 74,0 & 82,5 & 82,5 \\
\hline \multirow[t]{2}{*}{ Crespa } & 71,5 & 80,0 & 82,5 & 74,0 \\
\hline & \multicolumn{4}{|c|}{ AM 7 DAS $(\mathrm{cm})$} \\
\hline Lisa & $0,68^{\mathrm{NS}}$ & 0,70 & 0,95 & 0,95 \\
\hline \multirow{2}{*}{ Crespa } & 0,90 & 0,94 & 0,94 & 0,91 \\
\hline & \multicolumn{4}{|c|}{ AM 14 DAS $(\mathrm{cm})$} \\
\hline Lisa & $1,42 b^{*}$ & $1,74 \mathrm{a}$ & $1,94 \mathrm{a}$ & $1,91 \mathrm{a}$ \\
\hline \multirow{2}{*}{ Crespa } & $2,19 \mathrm{a}$ & $2,07 \mathrm{a}$ & $2,19 \mathrm{a}$ & $2,0 \mathrm{a}$ \\
\hline & \multicolumn{4}{|c|}{ AM 21 DAS $(\mathrm{cm})$} \\
\hline Lisa & $2,70 \mathrm{~b}$ & $3,30 \mathrm{a}$ & $3,38 \mathrm{a}$ & $3,21 \mathrm{a}$ \\
\hline \multirow{2}{*}{ Crespa } & $3,91 \mathrm{a}$ & $3,40 \mathrm{a}$ & $3,83 \mathrm{a}$ & $3,77 \mathrm{a}$ \\
\hline & \multicolumn{4}{|c|}{ AM 28 DAS $(\mathrm{cm})$} \\
\hline Lisa & $2,70 \mathrm{~b}$ & $3,30 \mathrm{a}$ & $3,38 \mathrm{a}$ & $3,21 \mathrm{a}$ \\
\hline \multirow[t]{2}{*}{ Crespa } & $3,91 \mathrm{a}$ & $3,40 \mathrm{a}$ & $3,83 \mathrm{a}$ & $3,77 \mathrm{a}$ \\
\hline & \multicolumn{4}{|c|}{ NF 7 DAS } \\
\hline Lisa & $1,82^{\mathrm{NS}}$ & 1,93 & 2,43 & 2,47 \\
\hline \multirow[t]{2}{*}{ Crespa } & 2,46 & 2,40 & 2,55 & 2,47 \\
\hline & \multicolumn{4}{|c|}{ NF 14 DAS } \\
\hline Lisa & $3,18^{\mathrm{NS}}$ & 3,25 & 3,31 & 3,25 \\
\hline \multirow[t]{2}{*}{ Crespa } & 3,41 & 3,42 & 3,52 & 3,36 \\
\hline & \multicolumn{4}{|c|}{ NF 21 DAS } \\
\hline Lisa & $3,88^{\mathrm{NS}}$ & 3,81 & 4,12 & 4,05 \\
\hline \multirow[t]{2}{*}{ Crespa } & 3,88 & 4,21 & 4,12 & 4,05 \\
\hline & \multicolumn{4}{|c|}{ NF 28 DAS } \\
\hline Lisa & $4,55^{\mathrm{NS}}$ & 4,52 & 4,63 & 4,55 \\
\hline \multirow[t]{2}{*}{ Crespa } & 4,38 & 4,25 & 4,30 & 4,41 \\
\hline & \multicolumn{4}{|c|}{$\mathrm{CRP}(\mathrm{cm})$} \\
\hline Lisa & $6,03 \mathrm{a}$ & $5,95 \mathrm{a}$ & $7,05 \mathrm{a}$ & $7,08 \mathrm{a}$ \\
\hline Crespa & $4,88 \mathrm{~b}$ & $4,57 \mathrm{a}$ & $5,06 \mathrm{~b}$ & $4,32 \mathrm{~b}$ \\
\hline
\end{tabular}

*Médias seguidas de mesma letra na coluna, para cada variável e período de avaliação, não diferem entre si pelo teste de Tukey $(\mathrm{p}<0,05) .{ }^{\mathrm{NS}}=$ diferença não significativa na análise de variância. ${ }^{*}$ Means followed by the same letter in the column, for each variable and evaluation period, did not differ among the Tukey test $(p<0.05) .{ }^{N S}=$ non-significant difference in analysis of variance. 
De maneira geral, em relação ao fator cultivares, a resposta ao condicionamento pode estar diretamente ligada às características genéticas, especialmente no que tange as quantidades e distribuição das substâncias de reservas armazenadas nas sementes, já que este fator relaciona-se diretamente com a velocidade de embebição e por consequência com a germinação (ZHAO et al., 2018; SORIANO et al., 2014) e emergência de plântulas.

\section{CONCLUSÃO}

A germinação de sementes de chicória aumenta linearmente com o aumento de doses de Ascophyllum nodosum via condicionamento de sementes.

O condicionamento de sementes de chicória com Ascophyllum nodosum contribui na produção de mudas, com incrementos na porcentagem de emergência de plantas aos sete dias após a semeadura, assim como na velocidade de emergência.

A reposta ao condicionamento de sementes de chicória com Ascophyllum nodosum está relacionada às especificidades de cada cultivar.

\section{REFERÊNCIAS BIBLIOGRÁFICAS}

ASHRAF, R.; SULTANA, B.; RIAZ, S.; MUSTAQ, M.; IQBAL, M.; NAZIR, A.; ATIF, M.; ZAFAR, Z. Fortification of phenolics, antioxidant activities and biochemical attributes of radish root by plant leaf extract seed priming. Biocatalysis and Agricultural Biotechnology, Amsterdam, v. 16, n. 1, p.115-120, 2018.

BATTACHARYYA, D.; BABGOHARI, M. Z.; RATHOR, P.; PRITHIVIRAJ, B. Seaweed extracts as biostimulants in horticulture. Scientia Horticulturae, Amsterdan, v. 196, n. 1, p.39-48, 2015.

BRASIL. MINISTÉRIO DA AGRICULTURA, PECUÁRIA E ABASTECIMENTO. SECRETARIA DE DEFESA AGROPECUÁRIA. Regras para análise de sementes. Brasília: Mapa/ACS, 2009. 398 p.

CARDOSO, A.I.I.; USTULIN FILHO, A.J. Produção de chicória em função de doses de nitrogênio e potássio aplicadas na fase de mudas. Horticultura Brasileira, Brasília, v. 31, n. 4, p.654-658, 2013.

DEMIRKAYA, M.; AYDIN, B.; ŞEKERCI, A. D.; GULSEN, O. Effects of Osmotic Conditioning Treatments of Lavender (Lavandula angustifolia) Seeds on Mean Germination Time and Germination Rate. International Journal of Secondary Metabolite, Denizili, v. 4, n. 3, p.418-422, 2017.

ELLIS, R. H.; BUTCHER, P. D. The effects of priming and 'natural' differences in quality amongst onion seed lots on the response of the rate of germination to temperature and the indentification of the characteristics under genotypic control. Journal of Experimental Botany, Oxford, v. 39, n.7, p.935-950, 1988. 
FERREIRA, R. L.; FORTI, V. A.; SILVA, V. N.; MELLO, S. C. Temperatura inicial de germinação no desempenho de plântulas e mudas de tomate. Ciência Rural, Santa Maria, v. 43, n. 7, p.1189-1195, 2013.

FOEKEL, E.; MATEUS, M. A. F.; MÓGOR, A. F.; BRUGNARA, E. C. Bioestimulantes aplicados às sementes e folhas de mudas de maracujazeiro-amarelo. Revista Cultura Agronômica, Ilha Solteira, v. 24, n. 2, p.135-148, 2015.

KHAN, W.; RAYIRATH, U. P.; SUBRAMANIAN, S.; JITHESH, M. N.; RAYORATH, P. Seaweed extracts as biostimulants of plant growth and development. Journal of Plant Growth Regulation, New York, v. 28, n. 4, p.386-399, 2009.

LOPES, H. M.; MENEZES, B. R. S.; SILVA, E. R.; RODRIGUES, D. L. Condicionamento fisiológico de sementes de cenoura e pimentão. Revista Brasileira de Agrociência, Pelotas, v. 17, n. 3-4, p.296-302, 2011.

MAGUIRE J. D. Speeds of germination-aid selection and evaluation for seedling emergence and vigor. Crop Science, Madison, v. 2, n. 1, p.176-177, 1962.

MARCOS-FILHO, J. Fisiologia de sementes de plantas cultivadas. 2. ed., Londrina: ABRATES, 2015. 660p.

MIRANSARI, M.; SMITH, D. L. Plant hormones and seed germination. Environmental and Experimental Botany, Elmsford, v. 99, n. 1, p.110-121, 2014.

NAKAGAWA, J. Testes de vigor baseados no desempenho das plântulas. In: KRZYZANOSKI, F. C; VIEIRA, R. D; FRANÇA NETO, J. B. (ed.) Vigor de sementes: conceitos e testes. Londrina: ABRATES, 1999. cap. 2, p. 21-24.

PAPARELLA, S.; ARAÚJO, S. S.; ROSSI, G.; WIJAYASINGHE, M.; CARBONERA, D.; BALESTRAZZI, A. Seed priming: state of the art and new perspectives. Plant Cell Reports, Berlin, v. 34, n. 8, p.1281-1293, 2015.

PROVASOLI, L.; CARLUCCI, A. F. Vitamins and growth regulators. In: STEWART, W. D. P. (Ed.). Algal physiology and biochemistry. London: Blackwell, 1974. cap. 27, p. 741-787.

SADEGI, H.; ROBATI, Z. Response of Cichorium intybus L. to eight seed priming methods under osmotic stress conditions. Biocatalysis and Agricultural Biotechnology, Taichung, v.4, n. 4, p.443-448, 2015.

SHARMA, A. D.; RATHORE, S. V. S.; SRINIVASAN, K.; TYAGI, R. K. Comparison of various seed priming methods for seed germination, seedling vigour and fruit yield in okra (Abelmoschus esculentus L. Moench). Scientia Horticulturae, Amsterdan, v. 165, n. 1, p.7581, 2014.

SILVA, C. B.; MARCOS FILHO, J.; JOURDAN, P.; BENETT, M. Performance of bell pepper seeds in response to drum priming with addition of 24-epibrassinolide. Hortscience, Alexandria, v. 50, n. 6, p.873-878, 2015.

SILVA, V. N.; DOTTO, L.; HAJAR, A. S.; BITTENCOURT, K. C.; STELlA, M. R. Production of Antirrhinum majus seedlings on different substrates and containers. Revista Científica, Jaboticabal, v. 45, n. 2, p.169-174, 2017. 
SIVRITEPE, H. O.; SIVRITEPE, N. Organic seed hydration-dehydration techniques improve seedling quality of organic tomatoes. Notulae Botanicae Horti Agrobotanici, Cluj-Napoca, v. 44, n. 2, p.399-403, 2016.

SORIANO, D.; HUANTE, P.; GAMBOA-DEBUEN, A.; OROZCO-SEGOVI, A. Effects of burial and storage on germination and seed reserves of 18 tree species in a tropical deciduous forest in Mexico. Oecologia, Berlin, v. 174, n. 1, p.33-44, 2014.

SOUZA NETA, M. L. Ação do bioestimulante na cultura do maxixeiro (Cucumis anguria L.) sob condições de estresse salino. 2016. 122 f. Dissertação (Mestrado em Fitotecnia) Universidade Federal Rural do Semi-Árido, Mossoró, 2016.

STIRK, W. A., NOVAK, M. S.; VAN STADEN, J. Cytokinins in macroalgae. Plant Growth Regulation, Dordrech, v. 41, n, 1, p.13-24, 2003.

WALly, O. S. D.; CRITCHLEY, A. T.; HILTZ, D.; CRAIGIE, X. H.; ZAHARIA, L. I.; ABRAMS, S.R.; PRITHIVIRAJ, B. Regulation of phytohormone biosynthesis and accumulation in Arabidopsis following treatment with commercial extract from the marine macroalga Ascophyllum nodosum. Journal of Plant Growth Regulation, New York, v. 32, n. 2, p.324-339, 2013.

ZHAO, Y.; HU, M.; GAO, Z.; CHEN, X.; HUANG, D. Biological mechanisms of a novel hydro-electro hybrid priming recovers potential vigor of onion seeds. Environmental and Experimental Botany, Elmsford, v. 150, n. 1, p.160-171, 2018. 